\title{
Relativistic time effects in financial dynamics
}

\author{
J.A. Tenreiro Machado
}

\begin{abstract}
Financial time series have a complex dy- namic nature. Many techniques were adopted having in mind standard paradigms of time flow. This paper explores an alternative route involving relativistic effects. It is observed that the measuring perspective influences the results and that we can have different time textures.
\end{abstract}

\section{Keywords}

Relativity;'Time series, Complex dynamics,

Financial analysis, Multidimensional scaling

\section{Introduction}

Financial time series (FTS) reflect the outcome of intricate relationships between several distinct agents such as economical, social, methodological and political processes $[1,18,23,24]$. There are many financial indices for capturing the characteristics and evolution of different markets and stock exchange institutions, but it is well known that, in general, they all have a fractal nature with variations difficult to preview [6, 10-17]. Different approaches have been proposed to analyse these objects in order to master the complex dynamics $[4,9,19,20,22]$, but all have in common the underlying undisputed concept of linear time flow. This paper studies the interplay between the evolution of FTS and the flow of time. The modern occidental assumption is that time is composed by a continuous linear succession of events and that we can define the concepts of "past", "present" and "future", producing what is often called as "time arrow". Three a priori clarifications need to be introduced at this stage. First, we shall not investigate if time has a continuous or a discrete nature. We shall adopt two alternative FTS, as supporting "leitmotif" for the experiments, that are sampled at daily basis. Therefore, the processing to be developed just uses data in the available (discrete) format; however, this procedure does not mean that a choice is made between continuous or discrete flows. Second, we do not intend to generalize the results up to the natural world. Both topics remain to be further investigated using more data from a larger set of sources and phenomena. In fact, we adopt the FTS merely because these objects are (loosely speaking) "free" from the laws of physics, being our only obligation to play the game of time evolution. We propose ourselves to adopt the "Gedankenexperiment" as popularized by Albert Einstein. We are talking of thought experiments, starting from hypothesis and concepts, and deriving logical consequences. Third, it is not intended to produce any type of estimation of financial nature. Our purpose is merely to take advantage of a complex non-smooth time series to investigate possible alternatives for conceiving the flow of time. There is a controversy about the texture 
of time and theories that range from the absence of the succession of time events (i.e., there is no such thing as "past", "present" and "future"), that time is reversed (i.e., the flow is "future", "present" and "past"), that time is circular, and many others $[2,5,7,21]$. In this paper we shall consider that time exists and that the FTS is embed in it; therefore, the existence of time and it evolution allows the existence of the FTS.

Bearing these ideas in mind this paper is organized as follows. Section 2 formulates the fundamental concepts. Section 3 develops the procedures and algorithms for estimating the relativistic time. Section 4 applies the concept of relativistic time in financial time series. Finally, Sect. 5 draws the main conclusions.

\section{Fundamental concepts}

We start by formulating our main postulate about time underlying the following experiments. We postulate that we have the common time where we are embedded and used to think of, and there are other manifestations of time not included directly in present day modelling formalism. In other words, we postulate that we have several distinct realizations of time (probably many) depending upon the perspective adopted when measuring a given phenomenon. Therefore, whether the out-coming time texture is linear or not, and whether it reveals some pattern, will depend on the particular phenomenon and the measure to be adopted. Let us recall the Einstein's relativistic experiments with passengers in the moving train. The railway transportation system is complicated, with many railroad switches and possible loops, commanded by a regional supervisor. The train has a carriage with no windows and the doors close perfectly, so that no external sounds reach the passengers. Furthermore, some sophisticated suspension systems prevents passengers from suffering acceleration forces. Under these conditions, passengers have no idea if they are in motion, or even the route they are taking at a given instant is unknown to them. If the railway supervisor, based on some private agenda or some choice of his own, decides to switch the train trajectory, then the resulting trip may have a strange spacial trajectory, possibly with passengers passing over the same places repeatedly, or coming back and forward in some parts of the railway. Now, let us suppose that some vague stimulus is capable of penetrating the carriage and reaches the passengers' senses. For example, some scent of flowers, such as roses, daisies, lilies and tulips, are sensed inside the carriage. Depending on the train velocity the smell variation can be fast, or, in some cases, slow. A careful passenger would have a rough estimate of his trip based on the incomplete information, since he has no guarantee that similar odours belong to the same place, or, for a fixed location, that they are not changing. Having completed the trip the passenger describes his experiences to a friend and persuades him to embark on the second trip. This time the railway regional supervisor decides to activate the railroad switches differently. At the end of this new trip passengers would have experimented a completely distinct succession of odours, even if the visited parts of the railway system are the same.

In order to avoid misleading terms, in the sequel we denote as "standard time" the present day concept integrating the universal space-time continuum. We denote as "relativistic time" the one estimated by means of some type of measure and algorithm, that can have a non-smooth flow and can be, eventually, multidimensional.

The conceptual experiments intend to guide us in defining the following concepts:

1. the train trip in space will be the analogous of the evolution along "standard time";

2 . the changing odours penetrating the carriage correspond to the FTS values;

3. the comparison and interpretation of odours, that depend on the passengers' sensitivity, represents measuring indices for comparing and analysing partial segments of the FTS;

4. the railway supervisor agenda and calculations may be interpreted as a meta-level decision making structure that governs the world market (may be the misty "Stock Market Intelligence" of stockholders).

To unravel the railway supervisor agenda and strange choices, we have only the vague succession of odours that depend on each passenger's sensitivity. Furthermore, odours sensed as similar will be connected in the passengers mind as being originated at the same place; however, that is not certain and they may correspond to different locations having the same type of flowers! Therefore, we have:

1. different measures and comparison algorithms (of the FTS segments in "standard time") produce pos- 


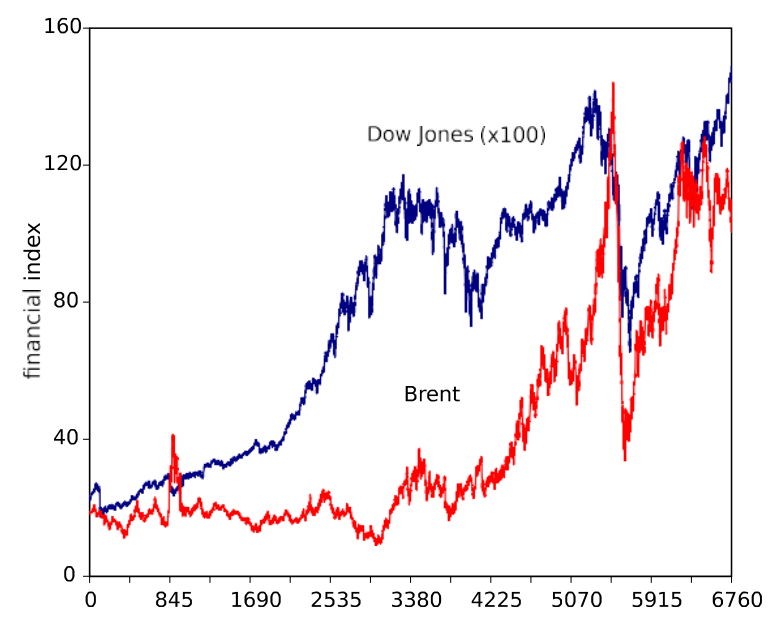

Fig. 1 Time evolution of the Dow Jones Industrial (DJI) Average and the Europe Brent Spot Price (FOB), from 18 May 1987 up to 12 April 2013 (6750 points)

sibly different estimates of the layouts (of "relativistic time");

2. the assumption that similar measures (of FTS segments in "standard time") correspond to similar periods (in "relativistic time") involves uncertainty.

\section{Estimating the relativistic time}

We adopt two FTS representing the Dow Jones Industrial Average (DJI) and the Europe Brent Spot Price (FOB) (in dollars per barrel). The daily data is available at the websites of the "Yahoo! Finance" and the "U.S. Energy Information Administration", respectively. The periodTstarting at 18 May 1987 and ending at 12 April 2013, is adopted in the sequel. Special days and holidays, which lead to some lack of data in the original time series, were estimated by simple interpolation of the neighbour values, so that all weeks include five days, making a total of 6750 points.

Figure 1 shows the time evolution of the Dow Jones Industrial Average and the Europe Brent Spot Price FOB over period $T$.

In both cases we divide the FTS into windows of $h=50$ points each, giving rise to $N=134$ objects, which are to be compared among themselves searching for possible similarities. This strategy introduces a discretization of time just for digital processing, therefore without excluding the hypothesis of continuous time. The Manhattan (city) distance $\left(d_{M}\right)$ or $L_{1}$-Norm, the Euclidean distance $\left(d_{E}\right)$ or $L_{2}$-Norm, and the Chebyshev distance $\left(d_{C}\right)$ or $L_{\max }-\mathrm{Norm}$, are compared. These measures are particular cases of the Minkowski distance given by

$$
d_{i j}=\left(\sum_{k=1}^{h}|x(k+i h)-x(k+j h)|^{\lambda}\right)^{\frac{1}{\lambda}}
$$

where $x(k+h)$ and $x(k j+h)$ are the $k$ th samples of signal $x$, for the time windows starting at $i h$ and $j h$, respectively, and parameter $\lambda \geq 1$ leads to $d_{M}, d_{E}$ and $d_{C}$ for $\lambda=1,2,+\infty$, respectively.

Also, the Jensen-Shannon divergence $\left(D_{J} S\right)$ is tested:

$$
d_{J S}=H\left(\frac{P_{i}+P_{j}}{2}\right)-\frac{1}{2}\left[H\left(P_{i}\right)+H\left(P_{j}\right)\right]
$$

where $H\left({ }^{\prime}\right)$ denotes the Shannon entropy, and $P_{i}$ and $P_{j}$ represent the probability distributions of the FTS windows $x(k i h)$ and $x(k j h), i, j \quad 1, \ldots, N$. For obtaining the histograms of relative frequency, 10 bins are adopted in the experiments.

\section{Relativistic time in financial time series}

In this section we perform two distinct types of experiments for the DJI and the FOB using the four previously defined measures. We start by subdividing the FTS into $N 134$ windows of $h 50$ points each. These objects are compared to each other and an $N N$ symmetric matrix $D$ of distances is built, where $d_{i j}$ represents the distance between the signal $x$ at the $i$ th and $j$ th time windows. Based on this matrix a Multidimensional Scaling (MDS) map is constructed. MDS is a computational and statistical technique that provides representation of the pattern of proximities among the set of objects. MDS plots points (i.e., the $N$ objects) that are perceived to be very similar/different are near to/far from each other in the map. The MDS map can have any number of dimensions $p$, although often $p 2$ and $p 3$ are adopted for the ease of visualization. In fact, MDS finds a set of vectors in a $p$-dimensional space such that the matrix of distances among the points with elements $\delta_{i j}$, approximates as closely as possible the input matrix $D$ according to a stress criterion $S \quad{ }_{i<j \quad}{ }_{N}\left(d_{i j} \delta_{i j}\right)^{2}$ to be minimized. It should be noted that MDS maps are not sensitive to scaling, translation or rotation and that usually axes have no units. A pseudo-code of the MDS algorithm is: 
Fig. 2 DJI versus relativistic time $\left(t_{1}, t_{2}\right)$ when applying the $d_{E}$. The point labels indicate the standard time

$i=0,1, \ldots, N-1$, for

$h=50$

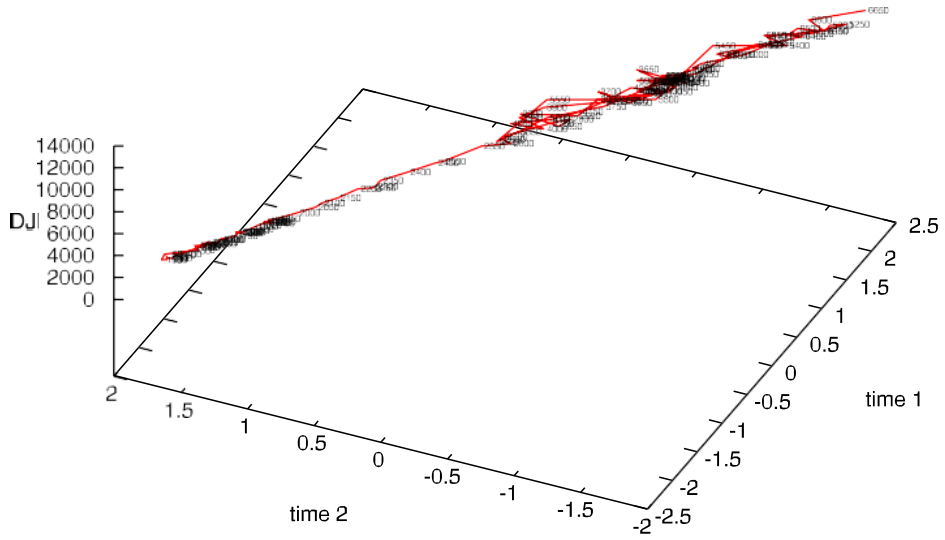

Fig. 3 Relativistic time $\left(t_{1}, t_{2}, t_{3}\right)$ locus for the DJI when applying the $d_{E}$. The point labels indicate the standard time

$i=0,1, \ldots, N-1$, for $h=50$

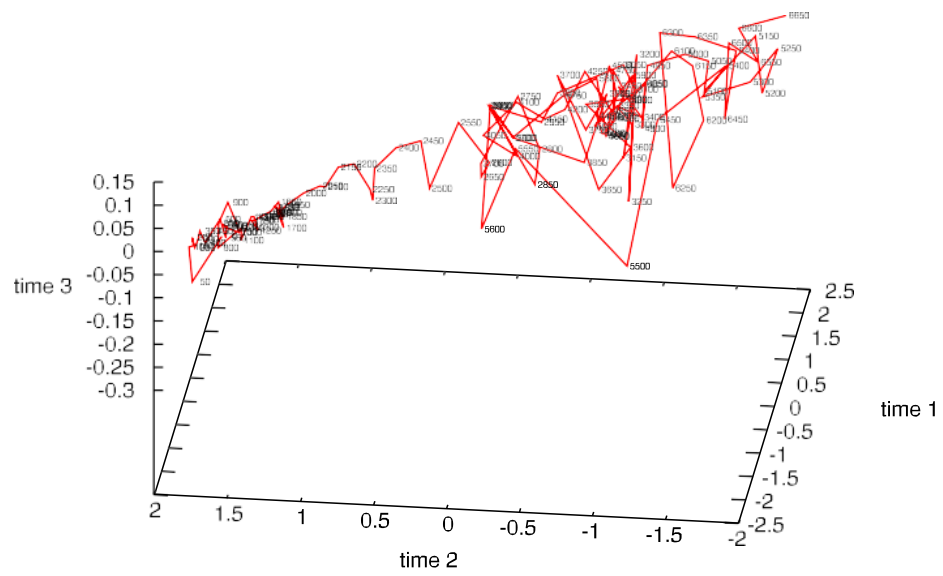

1. Calculate distances between all pairs of points, in the perspective of a given metric, producing the input matrix $D$.

2. Assign arbitrary coordinates to candidate points in the $p$-dimensional space.

3. Calculate distances between all pairs of points, leading to the output matrix

4. Compare input and output matrices by evaluating the stress function $S$. The smaller its value, the better.

5. Adjust coordinates of each candidate point in the direction that minimizes $S$.

6. Repeat Steps 3 through 5 until stress does not reduce further.

In the sequel the two sets of experiments differ from what amount of information is extracted from the MDS vector of coordinates. In a first case we consider $p \neq$ (i.e., we use the first two vectors of coordinates produced by MDS) as candidates for representing a two-dimensional "relativistic time". In the 3- dimensional chart, the third dimension is then used for representing the average of the FTS over the window of $h=50$ points. Each point is labelled with the value of the initial time $i, i=0,1, \ldots, N-1$, of that particular window. In a second case, we consider $p=3$ and we label each point with the initial time $i$. So, any information about the financial index is not depicted and only time before and after the processing is represented. We note again that we are comparing the values (i.e, periods of "relativistic time") of the FTS having in mind that similar values may eventually occur in similar places in a multidimensional relativistic time. Obviously, this leads to a relativistic time map where we have the "high" and the "low" values, similarly to the train passenger that draws a map of the locations of roses, daisies, lilies and tulips.

Figures $2-3$ and $4-5$ show the results for the DJI and FOB when applying measure $d_{E}$ for constructing matrix $D$. Figures 2 and 4 depict the financial index 
Fig. 4 FOB versus relativistic time $\left(t_{1}, t_{2}\right)$ when applying the $d_{E}$. The point labels indicate the standard time

$i=0,1, \ldots, N-1$, for $h=50$

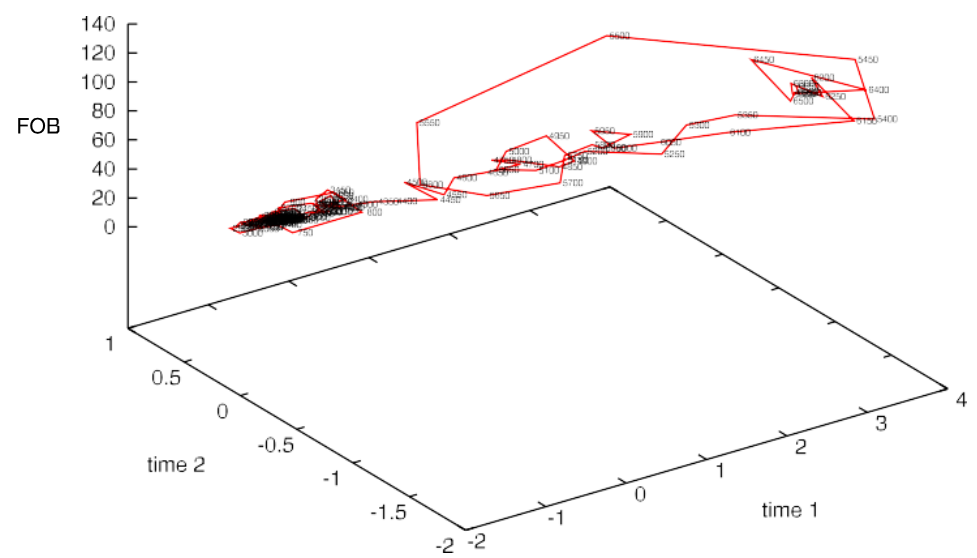

Fig. 5 Relativistic time $\left(t_{1}, t_{2}, t_{3}\right)$ locus for the FOB when applying the $d_{E}$. The point labels indicate the standard time $i=0,1, \ldots, N-1$, for $h=50$

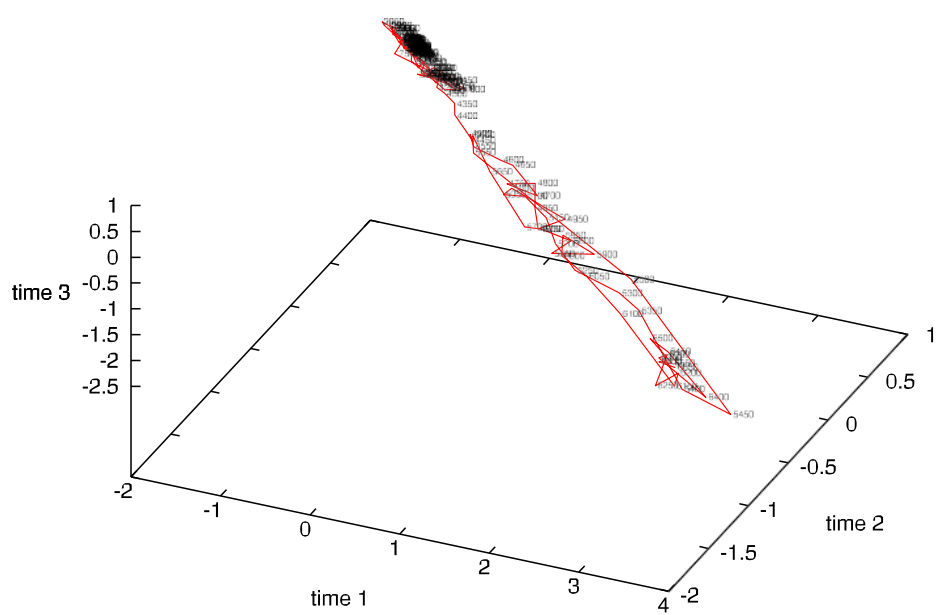

versus relativistic time $\left(t_{1}, t_{2}\right)$, while Figs. 3 and 5 represent merely the relativistic time $\left(t_{1}, t_{2}, t_{3}\right)$.

We observe that the changes in the values of the DJI and the FOB smooth considerably in the 2dimensional time locus. This effect is clarified in the 3-dimensional time locus where we observe the emergence of some time ripple that compensates for the reduction in the amplitude volatility of the financial indices. It is interesting to observe that on a different level the time locus has a global linear variation with the original time.

For the sake of limiting space we now present the charts for other distance measures, using merely the DJI.

Figures 6 and 7 show DJI versus relativistic time $\left(t_{1}, t_{2}\right)$ when applying the measures $d_{M}$ and $d_{C}$.

In both cases we observe a curvature of the locus contrary to what happened with the $d_{E}$.
The adoption of other expressions for distance lead to figures with small significance. For example, Fig. 8 shows DJI versus relativistic time $\left(t_{1}, t_{2}\right)$ and Fig. 9 depicts relativistic time $\left(t_{1}, t_{2}, t_{3}\right)$ locus for the DJI, with measure $d_{J S}$, revealing a chaotic structure. Some experiments with other indices revealed also figures apparently not well structured.

We have been using $h=50$ leading to $N=136$. Has this discretization of "standard time" any special effect upon the results? For investigating this topic we decided to repeat the procedure for $h=$ $20, N=340$ and measure $d_{E}$. Figure 10 shows DJI versus relativistic time $\left(t_{1}, t_{2}\right)$ and Fig. 11 depicts relativistic time $\left(t_{1}, t_{2}, t_{3}\right)$ locus for the DJI, with measure $d_{E}$. The labels are not included in order to provide a better visualization. Obviously we have a larger number of points, but globally we observe the same type of behaviour for the "relativistic time". 
Fig. 6 DJI versus relativistic time $\left(t_{1}, t_{2}\right)$ when applying the measure $d_{M}$. The point labels indicate the standard time $i=0,1, \ldots, N-1$, for $h=50$

Fig. 7 Relativistic time $\left(t_{1}, t_{2}, t_{3}\right)$ locus for the DJI when applying the measure $d_{C}$. The point labels indicate the standard time $i=0,1, \ldots, N-1$, for $h=50$

Fig. 8 DJI versus

relativistic time $\left(t_{1}, t_{2}\right)$ when applying the measure $d_{J S}$. The point labels indicate the standard time $i=0,1, \ldots, N-1$, for $h=50$
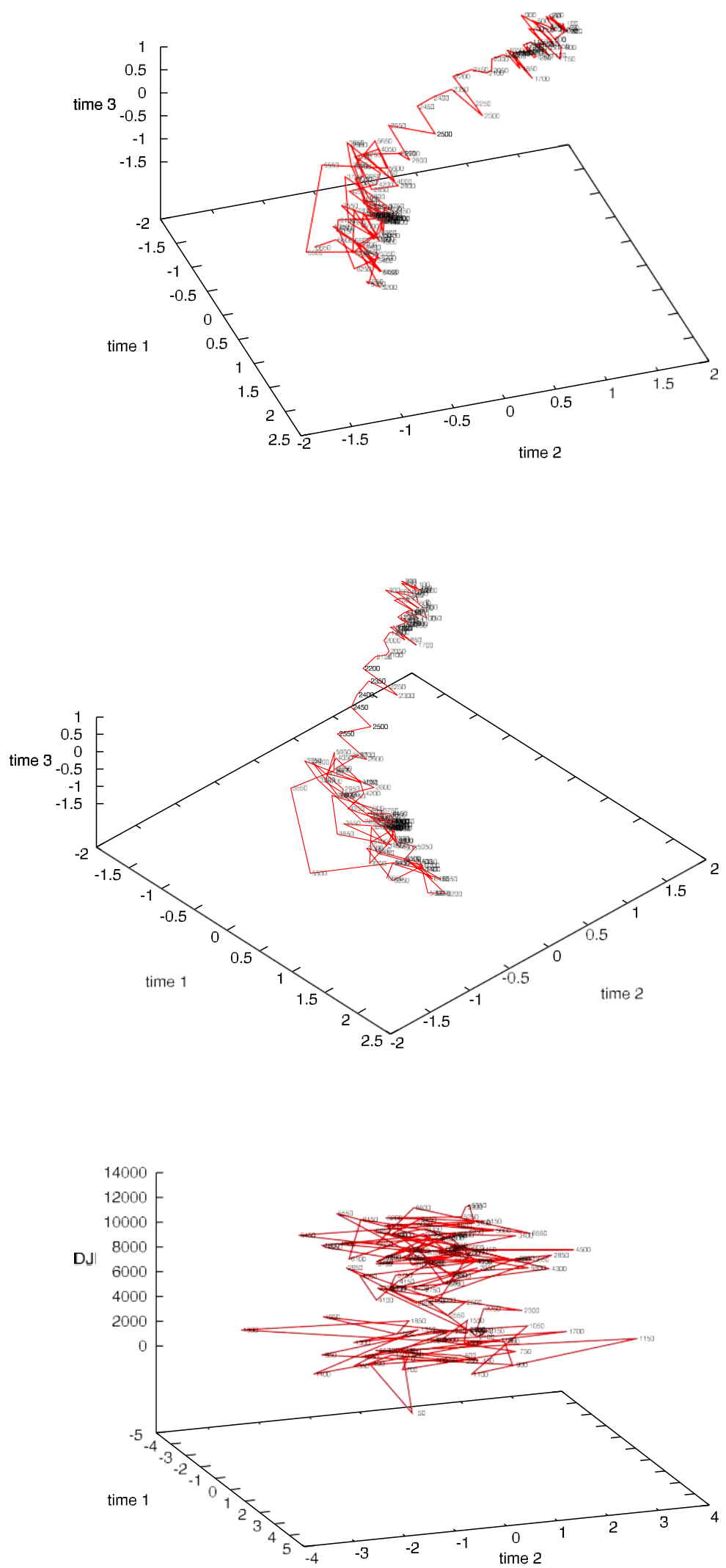
Fig. 9 Relativistic time $\left(t_{1}, t_{2}, t_{3}\right)$ locus for the DJI when applying the measure $d_{J S}$. The point labels indicate the standard time $i=0,1, \ldots, N-1$, for $h=50$

Fig. 10 DJI versus relativistic time $\left(t_{1}, t_{2}\right)$ when applying the measure $d_{E}$. The point labels indicate the standard time $i=0,1, \ldots, N-1$, for $h=20$

Fig. 11 Relativistic time $\left(t_{1}, t_{2}, t_{3}\right)$ locus for the DJI when applying the measure $d_{E}$. The point labels indicate the standard time $i=0,1, \ldots, N-1$, for $h=20$
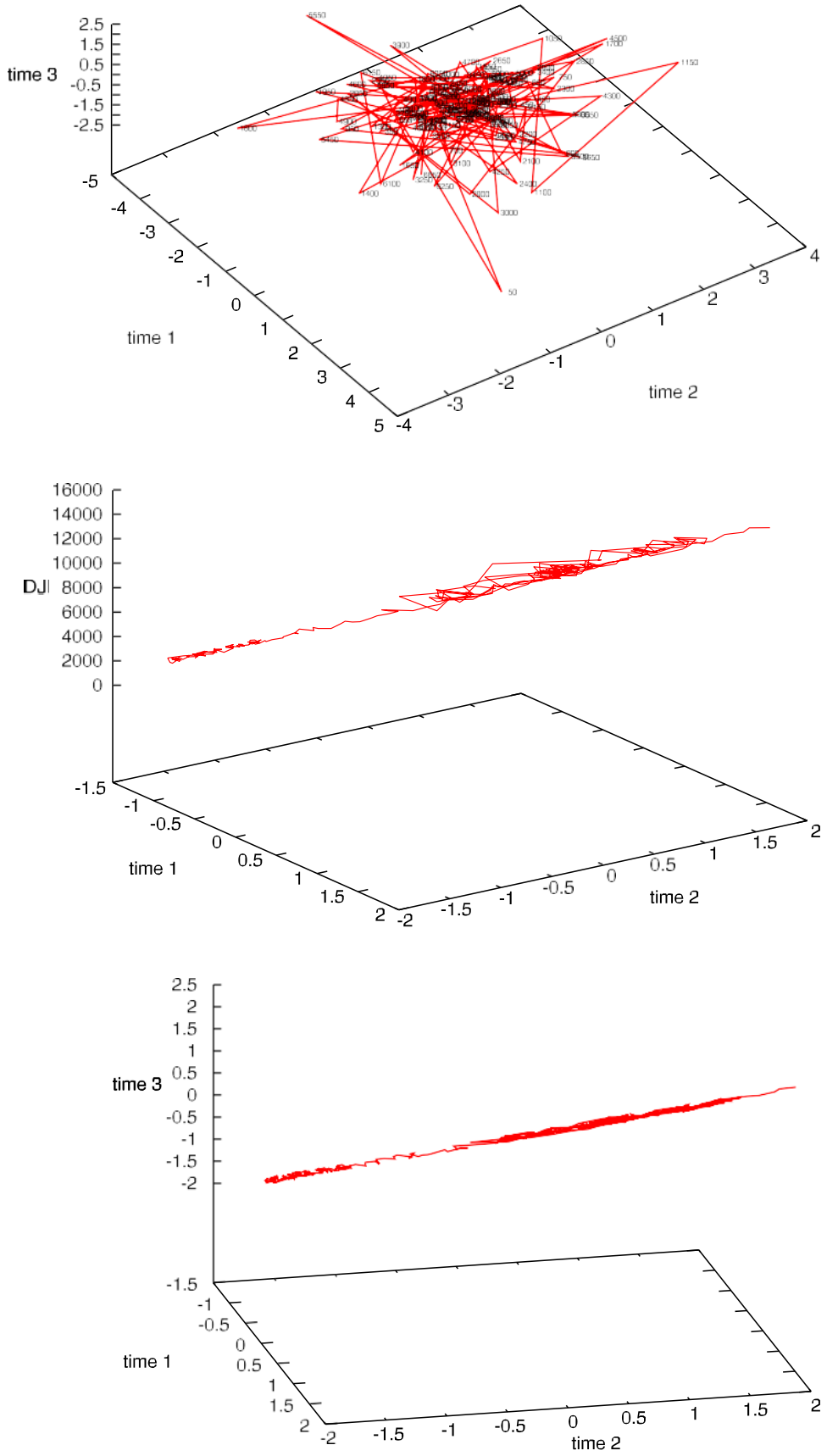

As mentioned previously, several measures produce charts of "relativistic time" of chaotic type. Therefore, we decided to investigate several possible measures. For example, measures based on the inner product (or cosine correlation) and Pearson Correlation lead to such chart and, therefore, are not depicted. However, cosine correlation) and Pearson Correlation lead to such chart and, therefore, are not depicted. However, a distinct distance measure leads to meaningful charts, as e.g. the one often denoted as "Camberra distance" $d_{B}[3,8]$, given by

$$
d_{i j}=\sum_{k=1}^{h}\left|\frac{x(k+i h)-x(k+j h)}{x(k+i h)+x(k+j h)}\right|^{\lambda}
$$


Fig. 12 DJI versus relativistic time $\left(t_{1}, t_{2}\right)$ when applying the measure $d_{B}, \lambda=1$. The point labels indicate the standard time $i=0,1, \ldots, N-1$, for $h=20$

Fig. 13 Relativistic time $\left(t_{1}, t_{2}, t_{3}\right)$ locus for the DJI when applying the measure $d_{B}, \lambda=1$. The point labels indicate the standard time $i=0,1, \ldots, N-1$, for $h=20$

Fig. 14 DJI versus relativistic time $\left(t_{1}, t_{2}\right)$ when applying the measure $d_{B}, \lambda=2$. The point labels indicate the standard time $i=0,1, \ldots, N-1$, for $h=20$
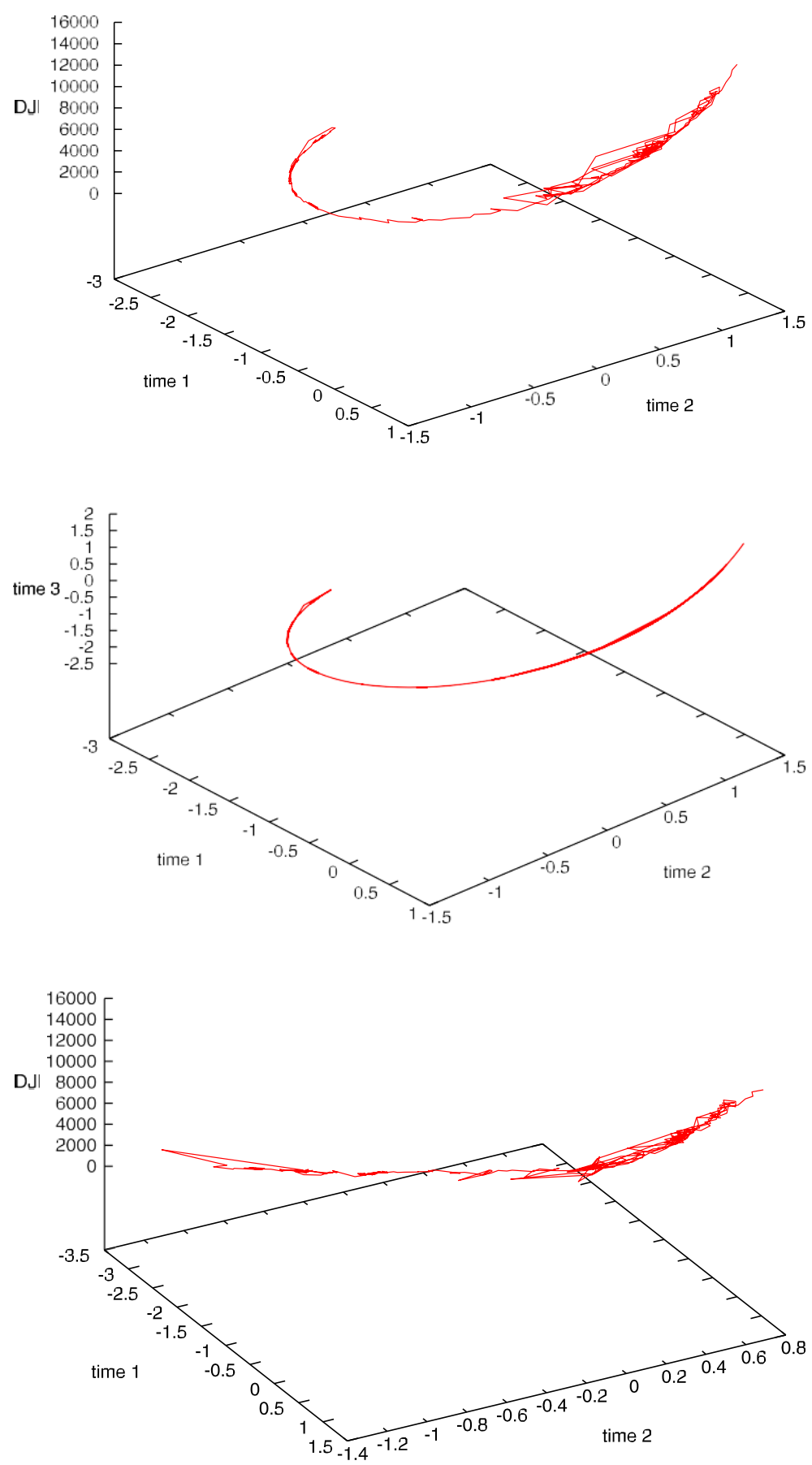

We observe a curvature for $\lambda=1$ (Figs. 12 and 13), but an almost linear behaviour for $\lambda=2$ (Figs. 14 and 15), and a reduced ripple in all cases. These results demonstrate that the type of measure has a strong influence on the texture that emerges for the "relativistic time".

\section{Conclusions}

The curvature of space-time related to energy and matter was explained by Einstein in the celebrated general theory of relativity. Nevertheless, time is generally accepted as a continuous linear flow and any 
Fig. 15 Relativistic time $\left(t_{1}, t_{2}, t_{3}\right)$ locus for the DJI when applying the measure $d_{B}, \lambda=2$. The point labels indicate the standard time $i=0,1, \ldots, N-1$, for $h=20$

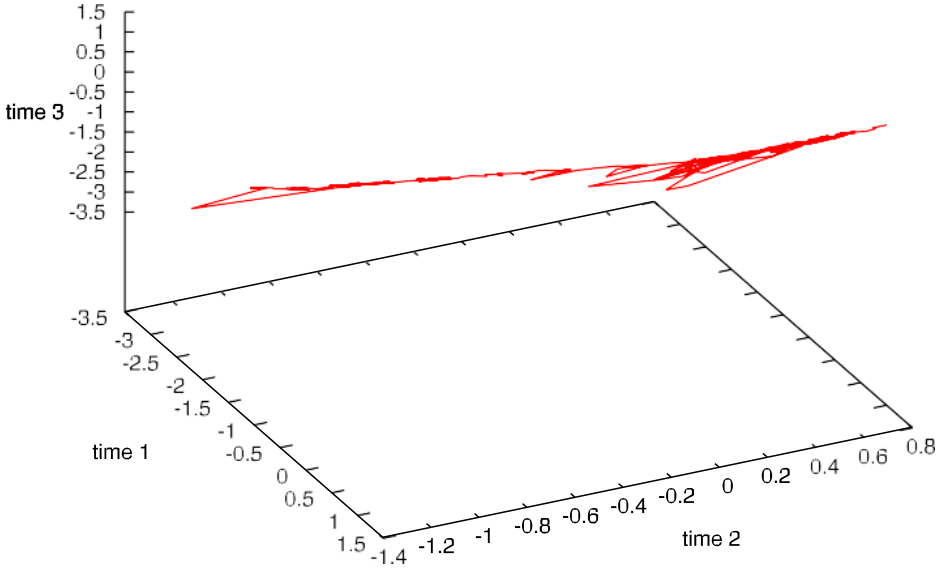

kind of anomaly is interpreted as the result of effects occurring in the phenomenon under analysis. In other words, the intangible nature of time favours strategies attacking the anomalies at source, that is at the measured variables. This paper explored an alternative idea, namely asking if some kind of volatility in the time flow can influence the variable under study. For avoiding the involvement of additional relationships with physics, financial time series are considered. In this case, the concepts of inertia, energy or momentum are not clear, but there is no doubt about its dependence upon time. Therefore, we do not need any laboratory equipment for testing the abstract financial index at different conditions and we can rely on thought experiments to analyse some hidden texture of time underlying the volatility of the index. The proposed approach was to construct a map based on similarities. We can debate that having similar values does not imply similar time. That is obvious, but anyone can testify distinct instants in their lifetime when some kind of resemblance makes them wonder about it. So, the paper simply proposes not to discard and to explore the possibility of having some (not all) of the similar values at similar time locations. If a computational method captures some time patterns, then errors will be viewed as an imperfect pattern. This postulate was tested for the DJI and the FOB under the measuring perspective of distinct indices. Furthermore, not only the evolution of the indices versus two components of the relativistic time, but also three components of relativistic time (only by themselves) were plotted. We verified that the measure influences the emerging pattern, with some distances producing clear charts, while others generating chaotic results. In our
"Gedankenexperiment" we verified that the sensitivity of the passenger's nose influences the determination of the map of roses, daisies, lilies and tulips, in what concerns its precision, but, more important, it seems that the layout of the gardens, through which the railway supervisor decides to direct the train, depends on the scheme that the passenger performs for comparing the fragrances. The author understands that some concepts are discussable and do not follow the main orthodoxy, but believes that this set of experiments is sufficiently solid for triggering new directions in exploring the texture of time.

\section{References}

1. Bischi, G.I., Chiarella, C., Gardini, L. (eds.): Nonlinear Dynamics in Economics, Finance and the Social Sciences. Springer, Berlin (2010)

2. Callender, C. (ed.): The Oxford Handbook of Philosophy of Time. Oxford University Press, New York (2011)

3. Cha, S.: Taxonomy of nominal type histogram distance measures. In: Proceedings of the American Conference on Applied Mathematics, Harvard, Massachusetts, USA, pp. 325-330 (2008)

4. Chen, W.C.: Nonlinear dynamics and chaos in a fractionalorder financial system. Chaos Solitons Fractals 36, 13051314 (2008)

5. Dainton, B.: Time and Space, 2nd edn. Acumen Publishing, Limited, Chesham (2001)

6. Duarte, F.B., Machado, J.A.T., Duarte, G.M.: Dynamics of the Dow Jones and the NASDAQ stock indexes. Nonlinear Dyn. 61(4), 691-705(2010)

7. Horwich, P.: Asymmetries in Time: Problems in the Philosophy of Science. MIT Press, Cambridge (1987)

8. M. M. Deza, E.D.: Encyclopedia of Distances. Springer, Berlin (2009)

9. Ma, J., Bangura, H.I.: Complexity analysis research of financial and economic system under the condition of three 
parameters' change circumstances. Nonlinear Dyn. 70(4), 2313-2326 (2012)

10. Machado, J.A.T.: Calculation of fractional derivatives of noisy data with genetic algorithms. Nonlinear Dyn. 57(12), 253-260(2009)

11. Machado, J.A.T., Duarte, G.M., Duarte, F.B.: Analysis of financial data series using fractional Fourier transform and multidimensional scaling. Nonlinear Dyn. 65(3), 235-245 (2011)

12. Machado, J.A.T., Duarte, G.M., Duarte, F.B.: Identifying economic periods and crisis with the multidimensional scaling. Nonlinear Dyn. 63(4), 611-622 (2011)

13. Machado, J.A.T., Duarte, G.M., Duarte, F.B.: Fractional dynamics in financial indexes. Int. J. Bifurc. Chaos 22(10), 1250249 (2012)

14. Machado, J.T., Duarte, F.B., Duarte, G.M.: Analysis of stock market indices through multidimensional scaling. Commun. Nonlinear Sci. Numer. Simul. 16(12), 4610$4618(2011)$

15. Machado, J.T., Duarte, F.B., Duarte, G.M.: Analysis of financial indices by means of the windowed Fourier transform. Signal Image Video Process. 6(3), 487-494 (2012)

16. Machado, J.T., Duarte, F.B., Duarte, G.M.: Power law analysis of financial index dynamics. Discrete Dyn. Nat. Soc. 2012, 120518(2012)
17. Machado, J.T., Duarte, G.M., Duarte, F.B.: Analysis of stock market indices with multidimensional scaling and wavelets. Math. Probl. Eng. 2012, 819503 (2012)

18. Meyers, R.A. (ed.): Complex Systems in Finance and Econometrics. Springer, New York(2010)

19. Ngounda, E., Patidar, K.C., Pindza, E.: Contour integral method for European options with jumps. Commun. Nonlinear Sci. Numer. Simul. 18(3), 478-492 (2013)

20. Piqueira, J.R.C., Mortoza, L.P.D.: Complexity analysis research of financial and economic system under the condition of three parameters' change circumstances. Commun. Nonlinear Sci. Numer. Simul. 17(4), 1690-1695 (2012)

21. Reichenbach, H.: The Direction of Time. University of California Press, New York(1991)

22. da Silva, S., Matsushita, R., Gleria, I., Figueiredo, A., Rathie, P.: International finance, Lévy distributions, and the econophysics of exchange rates. Commun. Nonlinear Sci. Numer. Simul. 10(4), 355-466 (2005)

23. Trippi, R.: Chaos \& Nonlinear Dynamics in the Financial Markets. Irwin, Scarborough (1995)

24. Vialar, T.: Complex and Chaotic Nonlinear Dynamics. Advances in Economics and Finance, Mathematics and Statistics. Springer, Berlin (2009) 